\title{
ENTOMOFAUNA ASOCIADA AL CULTIVO DE JAMAICA (HIBISCUS SABDARIFFA L.) EN EL MUNICIPIO DE CHIAUTLA DE TAPIA, PUEBLA
}

\author{
Betzabeth Cecilia PÉrez-Torres ${ }^{1}$, Agustín Aragón García², Néstor \\ BAUTiSTa Martínez ${ }^{3}$, Ana María TAPIA ROJAS ${ }^{2}$ \\ y Jesús Francisco LóPEZ-OLGUín 1,2 \\ 1Posgrado en Ciencias Ambientales. Instituto de Ciencias, \\ Benemérita Universidad Autónoma de Puebla. \\ 2Departamento de Agroecología y Ambiente, Instituto de Ciencias, BUAP. \\ ${ }^{3}$ Colegio de Postgraduados- Campus Montecillo Km 35.5 Carr. México Texcoco. 56230 Montecillo, \\ Texcoco Edo. de México, MÉXICO. e-mail: cs002445@siu.buap.mx, aragon@siu.buap.mx
}

Pérez-Torres, B. C., A. Aragón García, N. Bautista Martínez, A. M. Tapia Rojas y J. F. LópezOlguín. 2009. Entomofauna asociada al cultivo de jamaica (Hibiscus sabdariffa L.) en el municipio de Chiautla de Tapia, Puebla. Acta Zoológica Mexicana (n. s.), 25(2): 239-247.

RESUMEN. En Chiautla de Tapia, Puebla, mediante muestreos realizados cada 15 días de junio a septiembre del 2007, se efectuaron colectas en tres parcelas cultivadas con jamaica (Hibiscus sabdariffa L.), utilizando un aspirador entomológico manual y una red de golpeo. Se revisaron los tallos, nudos, entre nudos, hojas (haz y envés) y flores para hacer un diagnóstico de la entomofauna. Un total de 1,856 ejemplares fueron colectados, de los cuales se determinaron 17 especies, comprendidas en 6 órdenes, 11 familias y 19 géneros; mientras que 5 morfoespecies no fueron determinadas. Los insectos plaga que se encontraron ocasionando daños considerables al cultivo fueron: Atta mexicana, Sphenarium purpurascens, Melanoplus spp. y Aphis gossypii. Entre los insectos benéficos se encontró a la mosca de San Miguel, Allograpta sp (polinizadora), Hippodamia convergens y Polister apachus (especies depredadoras).

Palabras clave: depredador, diagnóstico, plagas.

Pérez-Torres, B. C., A. Aragón García, N. Bautista Martínez, A. M. Tapia Rojas \& J. F. LópezOlguín. 2009. Associated entomofauna with the jamaica cultivation (Hibiscus sabdariffa L.) in the municipality of Chiautla de Tapia, Puebla. Acta Zoológica Mexicana (n. s.), 25(2): 239-247.

ABSTRACT. In Chiautla de Tapia, Puebla, samplings were carried out at two-week intervals in three cultivated lots with jamaica (Hibiscus sabdariffa L.) from June to September in 2007. The collections were made by means of an entomologic aspirator in a manual way and with beating sheets. Stems, knots, inter-knots, leaves (front and back) and flowers were revised to make an entomofauna diagnosis. A total of 1,856 specimens were collected, of which, were determined 17 species, comprised in 6 orders, 11 families and 19 genera; while 5 morphospecies were not determined. The insects plague that were found causing serious damages to the crop were: Atta mexicana, Sphenarium purpurascens, Melanoplus spp. and Aphis gossypii, and amongst the beneficial insects are San Miguel's fly, Allograpta sp. (pollinator), Hippodamia convergens and Polister apachus (depredatory species).

Key words: Depredator, diagnosis, plagues.

Recibido: 16 /06/2007; aceptado: 5/02/2009. 


\section{INTRODUCCIÓN}

La jamaica (Hibiscus sabdariffa L.) es una planta originaria de la India que fue propagada a zonas tropicales y subtropicales de ambos hemisferios. En México, fue introducida por los españoles, se cultiva en regiones tropicales cálidas y semicálidas (Rojas, 1999); y actualmente en los estados de Campeche, Colima, Guerrero, Jalisco, Michoacán, Nayarit, Oaxaca, y Puebla.

Este cultivo, cada día esta tomando mayor importancia por productores y consumidores debido a sus diversos usos en la cocina, repostería, industria y fabricas textiles, utilizando el cáliz para elaborar aguas frescas, gelatinas, jaleas, mermeladas, ponche, refrescos, vinos entre otros. Las hojas y tallos se consumen en forma de ensaladas, además se utiliza como abono para las plantas, proporcionándole nutrientes al suelo y las semillas se han utilizado como sustituto afrodisíaco del café (Watt \& Breyer-Brandwijk, 1962). En la medicina herbolaria los cálices se utilizan para controlar el peso, es un remedio popular para los abscesos biliares, cáncer, debilidad, dispepsia, fiebre, hipertensión, neurosis, problemas del corazón, resfriados y tos. Actúa como laxante y previene el cáncer, también las hojas son utilizadas en forma de polvo para uso externo en la curación de heridas y llagas (García, 1995).

A pesar que la jamaica proporciona grandes beneficios, uno de los problemas que enfrenta el productor son las constantes pérdidas en su producción generados por daños ocasionados por insectos plagas, entre los cuales se pueden mencionar chapulines, gallinas ciegas, gusano soldado, hormiga arriera y pulgones. Debido a los bajos rendimientos del cultivo, se aplican métodos para combatirlos con productos químicos; sin embargo, el empleo irracional de los productos (uso de mezclas, productos no efectivos, aplicaciones persistentes, equipo mal calibrados, incremento en la frecuencia y dosis de las aplicaciones, entre otros factores), ocasionan una serie de problemas colaterales al ambiente y la salud de los seres vivos. Estos problemas son generados por no existir una planeación en el manejo y se dificulta aún más cuando se desconoce cuáles son las especies presentes en el cultivo. En el caso del cultivo de la jamaica, no se ha realizado ningún diagnóstico sobre los insectos asociados para determinar cuales son los insectos herbívoros y cuales los entomófagos y/o polinizadores.

Para realizar un manejo de las plagas con menos impacto al ambiente, es necesario obtener información precisa del problema, como la ubicación taxonómica de dichos organismos, conocer su biología, hábitos, ciclo de vida, fluctuación poblacional, enemigos naturales. En el caso de los insectos herbívoros es necesario estimar las pérdidas económicas que ocasionan al cultivo, a medida que se tenga mayor conocimiento de la especie a controlar, mayor será el éxito que se tenga en su manejo (Aragón et al. 2002).

En México es muy escasa la información que se ha generado sobre las plagas que interactúan con la jamaica, sólo se han reportado a Aphis gossypii, Atta mexicana y 
Maconellicocus hirsutus (Castro 2002, García 1995 y Pushpaveni et al. 1973). Debido a que no se conocen las especies de insectos asociadas a dicho cultivo en el municipio de Chiautla de Tapia, Puebla; se realizó esta investigación con el objetivo de determinar e identificar los insectos (benéficos y perjudiciales) asociados al cultivo de jamaica.

\section{MATERIAL Y MÉTODOS}

Se seleccionaron tres parcelas sembradas con jamaica en la zona agrícola de Chiautla de Tapia, Puebla, durante el ciclo agrícola (2003-2004). La primera parcela se ubicó en el campo Xocoatlaco, la segunda en la comunidad de Pilcaya y la tercera en San Juan de los Ríos del municipio antes indicado. Las prácticas que se realizaron fueron las que tradicionalmente se aplican a este cultivo; éstas consistieron en barbecho, surcado, deshierbe en forma manual al momento de la primera labor (que se realiza un mes después de la siembra), deshije, capado y cosecha, sin aplicación de agroquímicos. Las altitudes de los sitios de estudios varían desde 900 a $1800 \mathrm{~m} \mathrm{snm}$, con un clima cálido subhúmedo con lluvias en verano, temperatura media anual mayor de $22{ }^{\circ} \mathrm{C}$, precipitación promedio por mes menor de $60 \mathrm{~mm}$; con un periodo de sequía variable de 7 a 8 meses, que va de la mitad del mes de octubre a la mitad del mes de junio (INEGI, 2000). Cada parcela tuvo una superficie de $900 \mathrm{~m}^{2}$, donde se sembraron 900 plantas de jamaica repartidas en 36 surcos de 25 plantas cada uno. La distancia entre surcos y entre plantas fue de un metro.

Después de la emergencia de las plantas y hasta antes de la cosecha, en cada parcela se realizó un muestreo cada quince días. Para el muestreo se seleccionaron al azar 20 plantas utilizando una tabla de números aleatorios. Los insectos presentes se recolectaron de tres formas: utilizando un aspirador entomológico, revisión minuciosa de las plantas para colectar los insectos no voladores presentes en hojas (haz y envés) y tallos; y para colectar los insectos de vuelo rápido se utilizó una red de golpeo, en la parte central de la parcela.

Los insectos colectados fueron depositados en frascos de plástico y se etiquetaron de acuerdo a los datos pertinentes de colecta: municipio, localidad, fecha, número de planta y colector. Los frascos fueron llevados al laboratorio de Entomología del Departamento de Agroecología y Ambiente del Instituido de Ciencias de la Benemérita Universidad Autónoma de Puebla, donde se procesaron las muestras biológicas. Las larvas fueron fijadas en líquido Pampel por cinco días y posteriormente se pasaron a alcohol al $70 \%$ para su conservación; el $80 \%$ de los adultos se fijaron en alcohol al $70 \%$ y el resto de los ejemplares se colocaron en frascos letales a base de acetato de estilo, se montaron con alfiler entomológico y se etiquetaron. Para la determinación de especies se utilizó la guía de insectos plaga (Bautista, 2006), mientras que otros ejemplares se enviaron a especialistas para su determinación taxonómica. 


\section{RESULTADOS}

Un total de 1,856 ejemplares fueron colectados, de los cuales se determinaron 17 especies de 19 géneros pertenecientes a 11 familias de 6 órdenes (Cuadro 1), mientras que 5 morfoespecies no fueron identificadas. El orden de mayor abundancia fue Coleoptera con el $36 \%$ de las especies, seguido de Orthoptera con 21\%, Homoptera $16 \%$, Hymenoptera y Lepidoptera ambas $11 \%$, y Díptera 5\%.

Cuadro 1. Especies de insectos asociados al cultivo de jamaica en Chiautla de Tapia, Puebla.

\begin{tabular}{|c|c|c|c|}
\hline Nombre Común & Nombre científico & Familia & Orden \\
\hline Chapulín & $\begin{array}{l}\text { Sphenarium purpurascens } \\
\text { Trimerotropis pallidipennis } \\
\text { Chromacris versicolor } \\
\text { Melanophus } \mathrm{sp}\end{array}$ & Acrididae & Orthoptera \\
\hline Pulgón & $\begin{array}{l}\text { Macrosiphum sp } \\
\text { Aphis gossypii }\end{array}$ & Aphididae & Homoptera \\
\hline Mosquita blanca & Trialeurodes vaporariorum & Aleyrodidae & \\
\hline Pulga saltona & Disonycha melanocephala & Chrysomelidae & Coleoptera \\
\hline Catarinita & Diabrotica balteata & & \\
\hline Catarinita de la papa & Leptinotarsa decemlineata & & \\
\hline Mayate rayado & Acalymma trivittatum & & \\
\hline Catarinita & Hippodamia convergens & Coccinellidae & \\
\hline \multirow[t]{2}{*}{ Gallina ciega } & Phyllophaga ravida & & \\
\hline & P. obsoleta & Melolonthidae & \\
\hline Escarabajo rojo & Euphoria biguttata & & \\
\hline Avispa & Polistes apachus & Vespidae & Hymenoptera \\
\hline Hormiga arriera & Atta mexicana & Formicidae & \\
\hline Mosca de San Miguel & Allograpta sp & Syrphidae & Diptera \\
\hline Gusano soldado & Spodoptera exigua & Noctuidae & Lepidoptera \\
\hline Gusano de collar negro & Pholisora catullus & Hesperiidae & \\
\hline
\end{tabular}

Las especies que fueron determinadas se dividieron en dos categorías: insectos potencialmente perjudiciales y los insectos benéficos. Los insectos perjudiciales considerados como plagas de la jamaica son:

a) Atta mexicana, conocida comúnmente por los productores como hormiga arriera o cortadora de hojas, ocasiona considerables daños al follaje en infestaciones severas, su acción es más intensa cuando las plantas están en sus primeras etapas de desarrollo en donde llegan a defoliar hasta una superficie de $10 \mathrm{~m}^{2}$ en una noche. Los 
daños de esta plaga se caracterizan porque cortan las hojas del ápice hacia la base, dejando únicamente los tallos más gruesos; cuando la plaga no es controlada ocasiona pérdidas significativas en la producción.

b) Aphis gossypii y Macrosiphum sp (pulgones), especies que dañan las hojas del cultivo, se encontraron en el envés de las hojas de forma agregada, entre los meses de junio y julio. Los daños consisten en succionar la savia de los tejidos tiernos de las plantas, su acción se traduce en un debilitamiento de los órganos afectados, manifestándose por una reducción en el desarrollo, amarillamiento de hojas, cuando la planta está en sus primeras etapas de desarrollo. Estos daños se reflejan en una disminución de la producción y calidad de las flores. Adicionalmente el mayor daño ocasionado por estos vectores es la transmisión de enfermedades producidas por virus, ya que se observaron los síntomas característicos de éstas, como es el enchinamiento de las hojas que evita que las plantas se desarrollen normalmente.

c) Sphenarium purpurascens y Malanoplus sp (chapulines), de las cuales la primera especie ocasionó el mayor daño al follaje y estuvo presente entre los meses de agosto a octubre, los daños más fuertes se observaron cuando la planta alcanzó una altura de $50 \mathrm{~cm}$. En infestaciones fuertes se encontraron hasta 11 individuos por planta, población que puede llegar a causar una defoliación total y afectar severamente al cultivo.

d) Diabrotica balteata, los adultos son los causantes de los daños al alimentarse de las hojas del cultivo. El daño se caracteriza por las típicas perforaciones irregulares en las hojas llegando a consumir grandes cantidades de follaje, la población más abundante se encontró de julio a septiembre.

e) Spodoptera exigua (gusano soldado), los daños son ocasionados por las larvas al alimentarse de las hojas más tiernas de las plantas. Se presenta entre los meses de julio a septiembre, cuando las plantas tenían una altura de $20 \mathrm{~cm}$, que es cuando las plantas están en pleno crecimiento.

f) Phyllophaga ravida y $P$. obsoleta, estas especies son conocidas comúnmente como gallina ciega. Las larvas se alimentan de la raíz de la planta, lo que ocasiona una disminución en la cantidad de raíz formada y provoca una menor absorción de nutrientes, por lo que se ve afectado el crecimiento y desarrollo. Los daños más severos ocurren cuando las plantas son pequeñas, ya que éstas no se desarrollan adecuadamente, se marchitan o se deshidratan hasta que llegan a morir. Los daños se observaron entre los meses de julio y agosto.

Las especies que se encontraron ocasionando daños al cultivo de jamaica, se clasificaron de acuerdo al nivel de daño que se observó como; daño severo (***), cuando se presentó más del $60 \%$ de la planta dañada; daño moderado (**), entre el 30 y $60 \%$ de daño a la planta; y daño leve (*), daño menor al 30\% (Cuadro 2). 
Pérez-Torres et al.: Entomofauna de la jamaica en Chiautla de Tapia, Puebla

Cuadro 2. Especies de insectos que dañan o se alimentan del cultivo de jamaica.

\begin{tabular}{llll}
\hline Nombre Común & Especie & Nivel de daño & Orden \\
\hline Chapulín & Sphenarium purpurascens & $* *$ & Orthoptera \\
& Melanophus sp & $* *$ & \\
& Chromacris versicolor & $*$ & \\
& Trimerotropis pallidipennis & $*$ & Homoptera \\
Pulgón & Macrosiphum sp & $* *$ & \\
& Aphis gossypii & $* *$ & Coleoptera \\
Mosquita blanca & Trialeurodes vaporariorum & $*$ & \\
Pulga saltona & Disonycha melanocephala & $*$ & \\
Catarinita & Diabrotica balteata & $*$ & \\
Mayate rayado & Acalymma trivittatum & $*$ & Hymenoptera \\
Gallina ciega & Phyllophaga ravida & & Lepidoptera \\
Hormiga arriera & P. obsoleta & $*$ & \\
Gusano soldado & Atta mexicana & $* * *$ & \\
Gusano de collar negro & Spodoptera exigua & $*$ & \\
\hline
\end{tabular}

Nivel de daño: * leve, ** moderado y *** severos

Se pueden considerar como plagas primarias de la jamaica a A. mexicana, A. gossypii, Macrosiphun sp, S. purpurascens y Melanoplus sp., sin embargo por el desequilibrio ecológico que existe en la región por el mal manejo del cultivo, están como plagas potenciales las siguientes especies de insectos: Phyllophaga ravida, $P$. obsoleta, Diabrotica balteata, Trialeurodes vaporariorum, Spodoptera exigua y Pholisora catullus.

Los insectos benéficos encontrados en el cultivo se pueden agrupar en polinizadores y depredadores. Entre los insectos polinizadores del cultivo de jamaica está: Allograpta sp, conocida por los productores de la mixteca poblana como mosca de San Miguel por encontrarse en el mes de septiembre y se considera como el principal insecto polinizador de dicho cultivo. Otro de los insectos que contribuye en la polinización de este cultivo es Euphoria biguttata especie colectada en las flores durante la época de floración del cultivo que ocurre en septiembre y octubre,

De las especies que se encontraron depredando a insectos perjudiciales se encontró a Hippodamia convergens, tanto los adultos como las larvas de esta especie se encontraron alimentándose de Aphis gossypii y Macrosiphum sp. 


\section{DISCUSIÓN}

Estos resultados concuerdan con lo reportado por García (1995), quién cita a $A$. mexicana como plaga importante del follaje de la jamaica cuando las plantas son pequeñas.

El resultado obtenido sobre los pulgones, coincide con lo encontrado por Pushpaveni et al. (1973), quienes mencionaron que en la India el pulgón A. gossypii es una de las especies que mayor daño ocasiona al follaje de la jamaica y es considerado como una plaga de importancia económica. Sin embargo hace falta determinar las pérdidas reales de producción originadas por la enfermedad trasmitida por este insecto.

Los chapulines tienen una amplia distribución en los estados de Puebla y Tlaxcala (Anaya et al. 2000) y se ha reportado como una plaga importante en diversos cultivos incluyendo el amaranto (Aragón \& López-Olguín, 2001) y con esta investigación se confirma que es una plaga importante en este cultivo.

Las larvas de $D$. balteata son conocidas comúnmente como gusano alfilerillo y se ha reportado como plaga importante en maíz, sorgo, frijol, soya cacahuate, papa entre otros cultivos dañado el sistema radical de las plantas (Bautista, 2006), mientras que los adultos ocasionan daño en amaranto (Aragón \& López-Olguín, 2001).

El gusano soldado presenta una amplia distribución en el México, y es considerada plaga importante en chile, jitomate, cebolla, soya, arroz y algodonero (Bautista, 2006) y en este trabajo se reafirma su importancia como plaga para el sur del estado de Puebla.

Las especies de gallina ciega reportadas en este trabajo presentan una amplia distribución en México y se han reportando dañando severamente a diversos cultivos Morón (2003). No se ha reportado a estas especies dañando al sistema radical de la jamaica.

La catarinita $H$. convergens es un depredador importante, debido a que se ha reportado que su presencia disminuye considerablemente la población de pulgones y la regula en forma natural (Metcalf y Flint, 1984), por lo que se considera que puede ser utilizada en el control biológico de insectos plaga en Chiautla de Tapia, Puebla.

En el caso de los insectos entomófagos se encontraron las larvas de Allograpta sp alimentándose de pulgones y mosquita blanca, estos datos coinciden con lo reportado por López (1990), quien reportó que entre los depredadores de importancia para los pulgones se encuentran los coccinélidos del género Hippodamia y un sírfido del género Allograpta. Asimismo Ohashi \& Urdampilleta (2003), hacen mención que en Argentina se encontraron larvas del género Allograpta alimentándose del complejo de pulgones en el mismo cultivo, al igual que $H$. convergens. Así mismo, Barrios et al. (2004) en Acatzingo, Puebla, encontraron dos especies de importancia benéfica para el cultivo de la col, siendo la catarinita $H$. convergens y un sírfido del género Allograpta, los que estaban alimentándose de pulgones, mientras que la avispa 
Polistes apachus se encontró alimentándose de otros organismos, como adultos de moscas, coleópteros y lepidópteros, especies que no fueron posible identificar, por lo que fue difícil determinar su función en el cultivo.

AGRADECIMIENTOS. Agradecemos a la Fundación PRODUCE, Puebla por el financiamiento otorgado a través del proyecto "Adopción de tecnología para el control de plagas y enfermedades del cultivo de jamaica en el municipio de Chiautla de Tapia, Puebla", también a los productores del cultivo de la jamaica de Chiautla de Tapia, Puebla.

\section{LITERATURA CITADA}

Anaya, R. S., J. Romero N. \& V. López M. 2000. Manual de Diagnóstico para las especies de chapulines (Orthoptera: Acridoidea) del estado de Tlaxcala y estados adyacentes. Colegio de Postgraduados. México. $266 \mathrm{p}$.

Aragón, G. A. \& J. F. López-Olguín. 2001. Descripción y control de las plagas del amaranto. Benemérita Universidad Autónoma de Puebla. Puebla, México. 32 p.

Aragón, G. A., J. F. López-Olguín, A. M. Tapia R, N. Bonilla \& B. C. Pérez-Torres. 2002. Extractos vegetales una alternativa para el control de plagas del amaranto Amaranthus hypochondriacus L. Pp. 125-137. In: G. A. Aragón, J. F. López-Olguín y M. A. Tornero C. (Eds). Métodos para la Generación de Tecnología Agrícola de Punta. Publicación especial de la Benemérita Universidad Autónoma de Puebla, Pue. México.

Barrios, D. B., R. Alatorre R., H. G. Calyecac C. \& N. Bautista M. 2004. Identification and population fluctuation of cabbage pests (Brassica oleracea $\mathrm{cv}$. capitata) and their natural enemies in Acatzingo, Puebla, México. Agrociencia 38: 239-248.

Bautista, M. N. 2006. Insectos plaga, una guía ilustrada para su identificación. Colegio de Postgraduados. México. 113 p.

Castro, M. M. 2002. Análisis de comercialización de la jamaica al mercado de la Unión o Comunidad Europea. Tesis de Licenciatura. Universidad de Chapingo. División de Ciencias Econ. Adm. Chapingo, Edo de México. 65 p.

García, M., E. 1995. Efecto del deterioro de tres tamaños de semilla de jamaica (Hibiscus sabdariffa L.), bajo diferentes periodos de envejecimiento acelerado. Tesis de Licenciatura. Departamento de Fitotecnia. Universidad Autónoma Chapingo. Edo de México. 81 p.

INEGI. 2000. Síntesis geográfica nomenclátor y cartografía del estado de Puebla. Instituto Nacional de Estadística Geografía e Informática, México, D. F. 56 p.

López, A. B. 1990. Dinámica poblacional de Brevicoryne brassicae (L) (Homoptera: Aphididae) en brócoli en Chapingo, México. Tesis de Maestría en Ciencias. Centro de Entomología y Acarología. Colegio de Postgraduados. Montecillo, Edo de México. 82 p.

Metcalf, C. L. \& W. P, Willim. 1984. Insectos destructivos e insectos útiles, sus constumbres y su control. CECSA. México. 1208 p.

Morón, M. A. 2003. Diversidad, distribución e importancia de las especies de Phyllophaga Harris en México (Coleoptera: Melolonthidae). Pp. 1-27. In: Aragón, G. A., M. A. Morón y A. Marín J. (Eds). Estudios sobre Coleópteros del suelo en América. Publicación especial de la Benemérita Universidad Autónoma de Puebla. Puebla, México.

Ohashi, D. V. \& D. Urdampilleta J. 2003. Interacción entre insectos perjudiciales y benéficos en el cultivo de Tabaco de Misiones. Revista de Investigación Agropecuaria. 32: 113-124.

Pushpaveni, G. M., R. Rama \& P. Appa. 1973. Note on pests of mest in Andhra Pradesh. Jute Bulletion. 36:106-111. 
Rojas, P. J. P. 1999. Perspectivas de ampliación del mercado de la jamaica (Hibiscus sabdariffa L.), del estado de Guerrero. Tesis de licenciatura. División de Ciencias Económico Administrativas, Universidad Autónoma de Chapingo. Edo de México. 67 p.

Watt, J. M. \& M. G. Breyer-Brandwijk. 1962. The medicinal and poisonous plants of southern and eastern Africa. 2nd ed. E. \& S. Livingstone, Ltd., Edinburgh and London. 1457 p. 